\title{
The asymptotic near-tip solution for mode-III crack in steady growth in power hardening media
}

\author{
YU-CHEN GAO \\ Harbin Shipbuilding Engineering Institute, Harbin, China \\ XIAO-TI ZHANG \\ Institute of Mechanics, the Academy of Sciences, Beijing, China \\ KEH-CHIH HWANG \\ Tsinghua University, Beijing, China
}

(Received April 15, 1981)

\begin{abstract}
ABST RACT
It is shown that, for mode-III crack in steady growth in power hardening media the near-tip singularity is not of the power function type but is logarithmic. By consideration of the inner boundary layer in the neighborhood of the unloading boundary, the asymptotic near-tip solutions for stress and strain fields are obtained, which satisfy all the necessary relations of elastic-plastic continuum mechanics, including the condition of continuity of the plastic parts of strain-rates across the unloading boundary.
\end{abstract}

\section{Introduction}

The investigation of near-tip singularity fields is necessary for the development of fracture criteria and has become one of the central problems in fracture mechanics. The near-tip stress and strain fields for growing cracks are utterly different from those for stationary cracks. After some distance of growth (e.g., several times the size of the plastic zone), the near-tip fields approach a steady state, which remains time-invarient for an observer moving together with the crack-tip. As the terminal state of the growing crack, the crack in steady growth has been studied by many authors in recent years.

Chitaley and McClintock [1] obtained the solution for the steady state of growing modeIII cracks. Slepjan [2], Gao [3] Rice et al. [4] obtained independently the plane-strain solution for mode-I cracks in steady growth. For mixed mode-I-II cracks Gao and Hwang [5] show that the solution for steady state does not exist. All these solutions for elasticperfectly plastic materials show that the near-tip strain singularities for steadily growing cracks are much weaker than for stationary cracks. If we adopt as the fracture criterion a quantity related to the near-tip deformation (e.g., the critical strain a distance $r_{c}$ ahead of the tip, or the critical displacement a distance $r_{c}$ behind the tip), then the load required for steadystate growth (e.g., the value $J_{s s}$ of $J$-integral) will be much greater than for initiation of growth (e.g., the value $J_{\text {IC }}$ ).

However, most engineering materials are strain hardening. Dean and Hutchinson [6] have carried out the finite-element numerical analysis for plane strain mode-I cracks in steady growth in power hardening material. Adopting the fracture criterion of the critical open 
displacement, they found that the ratio $J_{s s} / J_{I C}$ is dependent upon the material exponent $n$. Hutchinson [7] pointed out that, since $J_{s s} / J_{\mathrm{IC}}$ increases with $n$, resistance curve predictions based on elastic-perfectly plastic calculations $(n=\infty)$ may well be highly unconservative.

The analytical solutions for the steady-state near-tip singularity fields in strain hardening materials are not many in number. Amazigo and Hutchinson [8] have obtained the solutions for mode-III and mode-I cracks in linear hardening materials. As pointed out by Hutchinson [6,7], singularity fields for a crack growing in a more realistic strain hardening solid such as power-hardening have been most elusive. Gao and Hwang [9] have made an attempt to obtain the singularity fields for plane strain mode-I cracks in a power hardening medium. Some further discussions have been made for mode-III cracks [10]. There is a deficiency in these solutions, namely, that the plastic part of the strain-rate does not vanish as $\theta$ (the polar angle centered at the tip) approaches the boundary between plastic loading and elastic unloading. This deficiency is more fully discussed in this paper for mode-III cracks after a brief introduction of the solution given in [10]. Taking into account the inner boundary layer near the unloading boundary, we obtain uniquely near-tip singularity fields without the above-mentioned deficiency.

\section{Basic equations and contiguity conditions}

In Fig. 1 the various zones in the $x, y$ plane are shown. Take the crack-tip as origin and assume the crack growing along the $x$-direction. $\Gamma_{A}$ is the borderline between the elastic zone I and the primary plastic zone II. $\Gamma_{B}$ denotes the boundary between the primary plastic zone II and the unloading wake zone III, and is called the unloading boundary. The unloading wake zone III is contiguous to the secondary plastic zone IV at $\Gamma_{D}$, which will be called the reloading boundary.

Denote the shear stress components $\tau_{x z}, \tau_{y z}$ simply by $\tau_{x}, \tau_{y}$, and the shear strain $\gamma_{x z}, \gamma_{y z}$ by $\gamma_{x}, \gamma_{y}$. The stress components can be expressed in terms of the stress function $\varphi$,

$$
\tau_{x}=\frac{\partial \varphi}{\partial y}, \quad \tau_{y}=-\frac{\partial \varphi}{\partial x} .
$$

The strain components can be split into the elastic and the plastic parts,

$$
\gamma_{x}=\gamma_{x}^{e}+\gamma_{x}^{p}, \quad \gamma_{y}=\gamma_{y}^{e}+\gamma_{y}^{p}
$$

The elastic part obeys the Hooke's law

$$
\gamma_{x}^{e}=\frac{\tau_{x}}{G}, \quad \gamma_{y}^{e}=\frac{\tau_{y}}{G}
$$

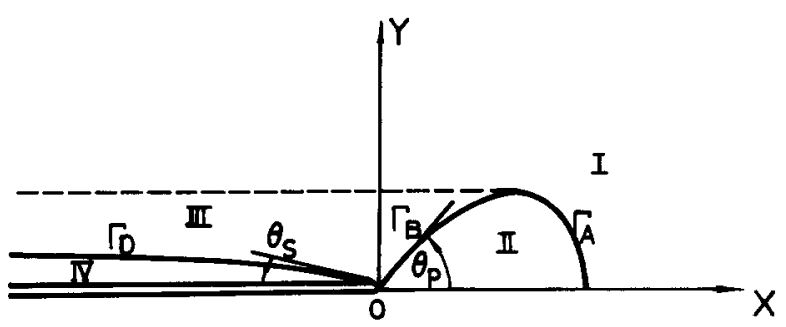

Figure 1. The various zones in the $x, y$ plane. 
where $G$ is the shear modulus. The plastic strain increments are determined by Reuss relations,

$$
d \gamma_{x}^{p}=\lambda \tau_{x} \mathrm{~d} a, \quad d \gamma_{y}^{p}=\lambda \tau_{y} \mathrm{~d} a .
$$

where $\mathrm{d} a$ denotes the crack-growth length, and $\lambda>0$. For steady state we have

$$
\frac{\partial}{\partial a}=-\frac{\partial}{\partial x} \text {. }
$$

From (2.4) and (2.5) we obtain

$$
\begin{array}{cl}
\frac{\partial \gamma_{x}^{p}}{\partial x}=-\lambda \tau_{x}, & \frac{\partial \gamma_{y}^{p}}{\partial x}=-\lambda \tau_{y}, \\
\gamma_{x}^{p}=\int_{x}^{x_{\mathrm{A}}} \lambda \tau_{x} \mathrm{~d} x, & \gamma_{y}^{p}=\int_{x}^{x_{\mathrm{A}}} \lambda \tau_{y} \mathrm{~d} x .
\end{array}
$$

From the relations between the strain components and the displacement $w$

$$
\gamma_{x}=\frac{\partial w}{\partial x}, \quad \gamma_{y}=\frac{\partial w}{\partial y}
$$

we can obtain the compatibility equation,

$$
\frac{1}{G} \Delta \varphi+\frac{\partial \gamma_{x}^{p}}{\partial y}-\frac{\partial \gamma_{y}^{p}}{\partial x}=0
$$

For the sake of convenience, we sometimes take the $x$-derivative of the compatibility equation (2.8), and then using (2.6), obtain

$$
\frac{1}{G} \frac{\partial}{\partial x} \Delta \varphi-\lambda \Delta \varphi-\frac{\partial \lambda}{\partial x} \frac{\partial \varphi}{\partial x}-\frac{\partial \lambda}{\partial y} \frac{\lambda \varphi}{\partial y}=0
$$

For strain hardening materials, we have

$$
\lambda=\left\{\begin{array}{llll}
-h \frac{\partial \tau}{\partial x}, & \text { when } & -\frac{\partial \tau}{\partial x} \geqslant 0 & \text { (loading). } \\
0, & \text { when } & -\frac{\partial \tau}{\partial x} \leqslant 0 & \text { (unloading). }
\end{array}\right.
$$

where $h$ is dependent upon the strain-hardening law, $h>0$, and $\tau$ is the resultant shear stress,

$$
\tau=\left(\tau_{x}^{2}+\tau_{y}^{2}\right)^{1 / 2}=\left\{\left(\frac{\partial \varphi}{\partial x}\right)^{2}+\left(\frac{\partial \varphi}{\partial y}\right)^{2}\right\}^{1 / 2} .
$$

For power hardening we have

$$
\gamma=\frac{\tau}{G}+c \tau^{n}
$$

where $c$ and $n$ are material constants and then

$$
h=c n \tau^{n-2}
$$

Some contiguity conditions must be satisfied at the boundary between neighboring zones. We assume that the boundary, $\Gamma$, is an arbitrary curve. We take the parallel-curves family of $\Gamma$ and their straight normals to be the coordinate lines, and represent the co- 
ordinate parameters by $s$ and $n$ (Fig. 2). Let $[\psi]_{\Gamma}$ denote the jump of $\psi$ across $\Gamma$, i.e. $[\psi]_{\Gamma}=\left.\psi\right|_{n=+0}-\left.\psi\right|_{n=-0}$. Then from the continuity of stresses, $\left[\tau_{n}\right]_{\Gamma}=\left[\tau_{s}\right]_{\Gamma}=0$, we obtain

$$
[\varphi]_{\Gamma}=0, \quad\left[\frac{\partial \varphi}{\partial n}\right]_{\Gamma}=0
$$

which will be called the first and the second contiguity conditions, respectively. From the continuity of the displacement $w$, i.e. $[w]_{\Gamma}=0$, the continuity of stresses (2.14) and hence of strains for strain hardening materials, we obtain the third contiguity condition,

$$
\left[\frac{\partial \gamma_{s}}{\partial n}\right]_{\Gamma}=0
$$

which, by use of (2.1), (2.3) and (2.6), can be written as

$$
\frac{1}{G}\left[\frac{\partial^{2} \varphi}{\partial n^{2}}\right]_{\Gamma}+\frac{\tau_{s}}{\cos \vartheta}[\lambda]_{\Gamma}=0 .
$$

Here $\vartheta$ is the angle from the $x$-axis to the normals of $\Gamma$. The third contiguity condition, (2.15) or (2.16), must be supplemented in addition to the first two contiguity conditions (2.14), only when we solve $\varphi$ from the $x$-derivative of the compatibility equation, i.e. (2.9), instead of the compatibility equation (2.8) itself.

For the unloading boundary, i.e. the boundary between the primary plastic zone and the unloading wake zone, an unloading condition should be supplemented. We have the following theorem:

THEOREM Let $\Gamma$ be an unloading boundary (Fig. 2). Then for steady state in strain hardening materials we have

$$
\begin{aligned}
& \left.\lambda\right|_{\Gamma(p)}=0 . \\
& \left.\frac{\partial \tau}{\partial x}\right|_{\Gamma(e)}=0 .
\end{aligned}
$$

where the subscripts $\Gamma(e)$ and $\Gamma(p)$ are used to denote the values on the boundary $\Gamma$ at the sides of unloading and loading zones, respectively.

For proof we refer to our previous paper [10]. Equation (2.17) is called the supplementary condition for the unloading boundary. As to Eqn. (2.18), it is a consequence of (2.16) and (2.17).

For the reloading boundary $\left(\Gamma_{\mathrm{D}}\right.$, Fig. 1), we have the following supplementary condition in addition to (2.14) and (2.16),

$$
\left.\tau\right|_{\Gamma_{\mathbf{D}}}(y)=\left.\tau\right|_{\Gamma_{\mathbf{B}}}(y)
$$

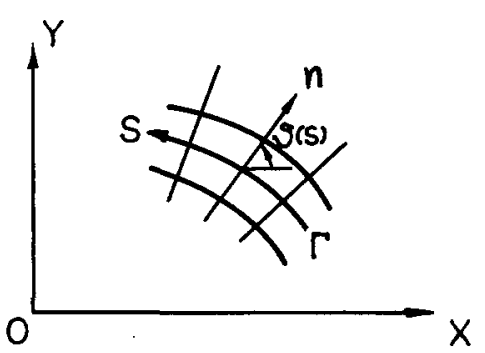

Figure 2. The curvilinear coordinates $(s, n)$ associated with the curve $\Gamma$. 


\section{The first version of asymptotic analysis - the power singularity}

We will first try the power expansion and, being confined only to the first order approximation, retain only the predominant term. Let $r, \theta$ denote polar coordinates and put

$$
\varphi=r^{1-\delta} f(\theta) \text {. }
$$

Then we have

$$
\tau_{r}=\frac{1}{r} \frac{\partial \varphi}{\partial \theta}=r^{-\delta} f^{\prime}(\theta), \quad \tau_{\theta}=-\frac{\partial \varphi}{\partial r}=-(1-\delta) r^{-\delta} f(\theta)
$$

From the $x$-derivative of the compatibility equation, (2.9), we obtain for the loading zone to the first order approximation

$$
\begin{aligned}
& \sin \theta \frac{1}{g} f^{\prime 2} f^{\prime \prime \prime}+\left(\delta \cos \theta+\frac{1}{2} \sin \theta \frac{g^{\prime}}{g}\right)\left[f^{\prime \prime}-n \delta(1-\delta) f\right] \\
& +f^{\prime}\left[\frac{1}{4}(n-3) \sin \theta \frac{g^{\prime 2}}{g^{2}}+\frac{1}{2}(1+\mu) \cos \theta \frac{g^{\prime}}{g}-\delta \sin \theta\right] \\
& +\sin \theta \frac{f^{\prime}}{g}\left\{f^{\prime \prime}\left[f^{\prime \prime}+(1-\delta)^{2} f\right]+(1-\delta)^{2} f^{\prime 2}\right\}=0,
\end{aligned}
$$

where

$$
g=f^{\prime 2}+(1-\delta)^{2} f^{2}, \quad \mu=(n-1) \delta .
$$

For the unloading zone, $\lambda \equiv 0$, we have then

$$
\sin \theta\left[(1-\delta)^{2} f^{\prime}+f^{\prime \prime \prime}\right]+\cos \theta(1+\delta)\left\{(1-\delta)^{2} f+f^{\prime \prime}\right\}=0 .
$$

The boundary conditions are

$$
f^{\prime}(0)=0, \quad f(\pi)=0 .
$$

The equation (3.3) with the boundary condition $f^{\prime}(0)=0$ can be solved only numerically. Since the coefficient of the highest derivative term in (3.3) vanishes at $\theta=0$, we are obliged to begin with some small angle $\theta$. The initial values $f\left(\theta_{0}\right), f^{\prime}\left(\theta_{0}\right)$ and $f^{\prime \prime}\left(\theta_{0}\right)$ at $\theta=\theta_{0}$ must be such that the value $f^{\prime \prime \prime}\left(\theta_{0}\right)$ can be computed from (3.3) with sufficient precision. For this purpose we use the following expressions from Taylor expansion,

$$
\begin{aligned}
& f\left(\theta_{0}\right)=f(0)+\frac{1}{2} \theta^{2} f^{\prime \prime}(0)+\frac{1}{24} \theta^{4} f^{\mathrm{IV}}(0)+\frac{1}{720} \theta^{6} f^{\mathrm{VI}}(0), \\
& f^{\prime}\left(\theta_{0}\right)=\theta f^{\prime \prime}(0)+\frac{1}{6} \theta^{3} f^{\mathrm{IV}}(0)+\frac{1}{120} \theta^{5} f^{\mathrm{VI}}(0), \\
& f^{\prime \prime}\left(\theta_{0}\right)=f^{\prime \prime}(0)+\frac{1}{2} \theta^{2} f^{\mathrm{IV}}(0)+\frac{1}{24} \theta^{4} f^{\mathrm{VI}}(0) .
\end{aligned}
$$

where $f^{\prime \prime}(0), f^{\mathrm{IV}}(0), f^{\mathrm{Vl}}(0)$ can be determined from (3.3) with the assumption of $f(\theta)$ being regular at $\theta=0$,

$$
\begin{aligned}
& f^{\prime \prime}(0)=n \delta(1-\delta) f(0), \\
& f^{\mathrm{IV}}(0)=n \delta(1-\delta)[n \delta(1-\delta)-2 n(1+\mu)(2+\mu)+2] f(0), \\
& f^{\mathrm{VI}}(0)=n \delta(1-\delta) f^{\mathrm{IV}}(0)-6[2 n(2 \delta+1)(1+\mu)-1]\left[f^{\mathrm{IV}}(0)-n \delta(1-\delta) f^{\prime \prime}(0)\right]- \\
& -36 n^{2} \delta\left[f^{\mathrm{IV}}(0)+(1-\delta)^{2} f^{\prime \prime}(0)\right]- \\
& -4 n^{2} \delta[6 n \delta(1+\mu)+7+4 n \delta(1-\delta)-8 n(1+\mu)(2+\mu)]\left[f^{\prime \prime}(0)+(1-\delta)^{2} f(0)\right]-
\end{aligned}
$$




$$
\begin{aligned}
& -24 n^{2}(n-3) \delta(1+\mu)^{2} f^{\prime \prime}(0)+ \\
& +4[12 n \delta(1+\mu)+1+n \delta(1-\delta)-2 n(1+\mu)(2+\mu)] f^{\prime \prime}(0)- \\
& -4 n(1+\mu)\left\{n \delta(1-\delta)(1+\mu)-2 n(1+\mu)^{2}(2+\mu)-(1+\mu)+3 n \delta(1-\delta)^{2}+\right. \\
& \left.+[1+(4 n-1) \delta][n \delta(1-\delta)-2 n(1+\mu)(2+\mu)+2]+6 n \delta(1+\mu)^{2}\right\} f^{\prime \prime}(0) .
\end{aligned}
$$

We have tried values of $\delta$ in the interval between 0.5 and 0.015 , and integrated (3.3) step by step from a small $\theta_{0}$. In our numerical calculation nowhere $\operatorname{did} f^{\prime}(\theta)$ happen to be zero (except at $\theta=0$ ) and nowhere did unloading take place. Thus we could adhere to (3.3) straight to the crack flank $\theta=\pi$. But for no value of $\delta \operatorname{did} f(\pi)$ happen to be zero. So we conclude that there are no existing solutions with power singularities of the form (3.1).

\section{The second version of asymptotic analysis - the logarithmic singularity [10]}

We assume the solution with logarithmic singularities of the form

$$
\varphi=r\left(\ln \frac{A}{r}\right)^{\alpha} \sum_{n=0}^{\infty} f_{n}(\theta)\left(\ln \frac{A}{r}\right)^{-n}, \quad f_{0}(\theta) \neq 0,
$$

where $A$ is a constant indeterminate within the scope of asymptotic analysis. Then the stress components $\tau_{r}, \tau_{\theta}$ and the resultant stress $\tau$ can be expanded as follows,

$$
\left.\begin{array}{rl}
\tau_{r} & =\frac{1}{r} \frac{\partial \varphi}{\partial \theta}=\left(\ln \frac{A}{r}\right)^{\alpha} \sum_{n=0}^{\infty} \tau_{r n}(\theta)\left(\ln \frac{A}{r}\right)^{-n}, \\
\tau_{\theta} & =-\frac{\partial \varphi}{\partial r}=\left(\ln \frac{A}{r}\right)^{\alpha} \sum_{n=0}^{\infty} \tau_{\theta n}(\theta)\left(\ln \frac{A}{r}\right)^{-n},
\end{array}\right\}
$$

where

$$
\begin{aligned}
& \tau_{r n}(\theta)=f_{n}^{\prime}, \quad(n \geqslant 0), \\
& \tau_{\theta 0}(\theta)=-f_{0}, \quad \tau_{\theta n}(\theta)=-f_{n}+(\alpha-n+1) f_{n-1}, \quad(n \geqslant 1), \\
& K_{0}(\theta)=f_{0}^{2}+f_{0}^{\prime 2}, \\
& K_{1}(\theta)=2 f_{0}^{\prime} f_{1}^{\prime}+2 f_{0}\left(f_{1}-\alpha f_{0}\right) .
\end{aligned}
$$

The prime " " is used to denote the derivative against $\theta$, if not otherwise stated. After some trials we come to select finally

$$
\alpha=\frac{2}{n-1} \text {. }
$$

Then from (2.10) and (2.13) we obtain

$$
\lambda=\frac{1}{r}\left(\ln \frac{A}{r}\right)^{2} \sum_{n=0}^{\infty} \lambda_{n}(\theta)\left(\ln \frac{A}{r}\right)^{-n},
$$

where

$$
\begin{aligned}
& \lambda_{0}(\theta)=\frac{1}{2} n c K_{0}^{(n-3) / 2} K_{0}^{\prime} \sin \theta, \\
& \lambda_{1}(\theta)=\frac{1}{2} n c K_{0}^{(n-5) / 2}\left\{\frac{1}{2}(n-3) K_{0}^{\prime} K_{1} \sin \theta+K_{0}\left(K_{1}^{\prime} \sin \theta+2 \alpha \cos \theta \mathrm{K}_{0}\right)\right\}
\end{aligned}
$$


For the $x$-derivative of the compatibility equation (2.9), in plastic loading zones (II or IV, Fig. 1) the first three asymptotic expansions will be the following,

$$
\begin{aligned}
& {\left[\lambda_{0}(\theta) f_{0}^{\prime}(\theta)\right]^{\prime}=0 .} \\
& \left(\lambda_{0} f_{1}^{\prime}\right)^{\prime}+\left(\lambda_{1} f_{0}^{\prime}\right)^{\prime}-\alpha n f_{0} \lambda_{0}=0 .
\end{aligned}
$$

and

$$
\begin{aligned}
& \left\{\left(\lambda_{0} f_{2}^{\prime}+\lambda_{1} f_{1}^{\prime}+\lambda_{2} f_{0}^{\prime}\right)^{\prime}+(1-\alpha n)\left(\lambda_{0} f_{1}+\lambda_{1} f_{0}\right)-\alpha(1-\alpha n) \lambda_{0} f_{0}\right\}+ \\
& +\frac{1}{G}\left\{\left(f_{0}^{\prime \prime \prime}+f_{0}^{\prime}\right) \sin \theta+\left(f_{0}^{\prime \prime}+f_{0}\right) \cos \theta\right\}=0
\end{aligned}
$$

Due to $f_{0}^{\prime}(0)=0$ for zone II and $\lambda_{0}(\pi)=0$ for zone IV (Fig. 1), from (4.6), (4.10) and (4.12) we have both zones

$$
\begin{aligned}
& \lambda_{0}(\theta)=0 . \\
& K_{0}^{\prime}(\theta)=2 f_{0}^{\prime}(\theta)\left\{f_{0}^{\prime \prime}(\theta)+f_{0}(\theta)\right\}=0 .
\end{aligned}
$$

Now we turn to the solution for each zone.

1. The primary plastic zone II (Fig. 1), $0 \leqslant \theta<\theta_{p}$. Take as the solution of (4.16)

$$
f_{0}(\theta)=\text { const }=-F \text {. }
$$

Then (4.4)-(4.6) will give

$$
\left.\begin{array}{l}
\tau_{r 0}(\theta)=0, \quad \tau_{\theta 0}(\theta)=F, \quad K_{0}(\theta)=F^{2}, \\
\tau_{x 0}(\theta)=-F \sin \theta, \quad \tau_{y 0}(\theta)=F \cos \theta .
\end{array}\right\}
$$

Equation (4.13) is satisfied identically, and (4.14) becomes

$$
\left\{\lambda_{1}^{*}(\theta) f_{1}^{* \prime}(\theta)\right\}^{\prime}+(\alpha+1) \lambda_{1}^{*}(\theta)-\frac{1}{S} \cos \theta=0
$$

where the following nondimensional quantities are introduced,

$$
\begin{aligned}
& S=c F^{n-1} G, \quad f_{1}^{*}(\theta)=\frac{1}{F} f_{1}(\theta), \\
& \lambda_{1}^{*}(\theta)=\frac{1}{c F^{n-1}} \lambda_{1}(\theta)=-n\left\{f_{1}^{*^{\prime}}(\theta) \sin \theta-\alpha \cos \theta\right\} \geqslant 0 .
\end{aligned}
$$

Here in the derivation of (4.21), Eqns. (4.7) and (4.11) have been used. From the Reuss relations (2.6) and using (4.2), (4.9), (4.15) and (4.18), we obtain the asymptotic expressions for plastic strains in zone II,

$$
\begin{aligned}
& \gamma_{x}^{p}=\left(\ln \frac{A}{r}\right)^{\alpha+1} \sum_{n=0}^{\infty} \gamma_{x n}^{p}(\theta)\left(\ln \frac{A}{r}\right)^{-n}, \\
& \gamma_{y}^{p}=\left(\ln \frac{A}{r}\right)^{\alpha+2} \sum_{n=0}^{\infty} \gamma_{y n}^{p}(\theta)\left(\ln \frac{A}{r}\right)^{-n} .
\end{aligned}
$$

where

$$
\gamma_{x 0}^{p}(\theta)=-c F^{n} \int_{0}^{\theta} \lambda_{1}^{*}(\theta) \mathrm{d} \theta, \quad \gamma_{y 0}^{p}(\theta)=c F^{n} .
$$


The integral appearing in (4.23) for $\theta=\theta_{p}$ can be obtained by integrating (4.19) and taking into account the condition of symmetry $f_{1}^{*^{\prime}}(0)=0$,

$$
\int_{0}^{\theta_{p}} \lambda_{1}^{*}(\theta) \mathrm{d} \theta=\frac{1}{\alpha+1}\left\{\frac{1}{S} \sin \theta_{p}-\lambda_{1}^{*}\left(\theta_{p}\right) f_{1}^{* \prime}\left(\theta_{p}\right)\right\} .
$$

2. The unloading wake zone III (Fig. 1). In the zone III the compatibility equation (2.8) reduces to

$$
\frac{1}{G} \Delta \varphi+\frac{\mathrm{d} y_{x}^{p}}{\mathrm{~d} y}=0 .
$$

Assume the asymptotic expansion for the plastic strains in the zone III in the form,

$$
\left.\begin{array}{l}
\gamma_{y}^{p}=\left(\ln \frac{A}{y}\right)^{\alpha+1} \sum_{n=0}^{\infty} a_{n}\left(\ln \frac{A}{y}\right)^{-n}, \\
\gamma_{y}^{p}=\left(\ln \frac{A}{y}\right)^{\alpha+2} \sum_{n=0}^{\infty} b_{n}\left(\ln \frac{A}{y}\right)^{-n} \cdot
\end{array}\right\}
$$

From the continuity of plastic strains across unloading boundary $\Gamma_{\mathrm{B}}\left(\theta=\theta_{p}\right.$, Fig. 1) and by comparison of (4.22) and (4.26), we obtain

$$
a_{0}=\gamma_{x 0}^{p}\left(\theta_{p}\right)=-c F^{n} \int_{0}^{\theta_{p}} \lambda_{1}^{*}(\theta) \mathrm{d} \theta, \quad b_{0}=c F^{n} .
$$

Substitution of (4.1) and (4.26) into (4.25) gives the first asymptotic expression of the compatibility equation (4.25),

$$
\frac{1}{G}\left[f_{0}^{\prime \prime}(\theta)+f_{0}(\theta)\right]-(\alpha+1) \frac{a_{0}}{\sin \theta}=0 .
$$

Its solution is

$$
f_{0}(\theta)=G a_{0}(\alpha+1)\left[A_{1} \sin \theta+B_{1} \cos \theta+f_{0}^{*}(\theta)\right],
$$

where

$$
f_{0}^{*}(\theta)=\sin \theta \ln (\sin \theta)-\left(\theta-\frac{\pi}{2}\right) \cos \theta .
$$

Then (4.4)-(4.6) will give the stresses.

3. The secondary plastic zone IV (Fig. 1), $\pi-\theta_{s}<\theta \leqslant \pi$. Noticing the free crack surface condition $\tau_{\theta 0}(\pi)=-f_{0}(\pi)=0$ and the supplementary condition (2.19) for the reloading boundary $\Gamma_{\mathrm{D}}$, we take as solution of (4.16)

$$
f_{0}(\theta)=F \sin \theta .
$$

And (4.4)-(4.6) will then give

$$
\left.\begin{array}{l}
\tau_{r 0}(\theta)=F \cos \theta, \quad \tau_{\theta 0}(\theta)=-F \sin \theta, \quad K_{0}(\theta)=F^{2}, \\
\tau_{x 0}(\theta)=F, \quad \tau_{y 0}(\theta)=0 .
\end{array}\right\}
$$

Noting (4.15), we obtain the solution of the second asymptotic expansion of the compatibility equation (4.13),

$$
\lambda_{1}(\theta)=\frac{b}{\cos \theta} .
$$


Substituting (4.32) and (4.33) into the Reuss relations (2.6) and noticing the continuity of plastic shear strains across the reloading boundary $\Gamma_{\mathrm{D}}\left(\theta=\pi-\theta_{\mathrm{s}}\right.$, Fig. 1), we obtain the constant $b=0$, and the plastic shear strains for zone IV,

$$
\left.\begin{array}{l}
\gamma_{x}^{p}=\left(\ln \frac{A}{r}\right)^{\alpha+1} \sum_{n=0}^{\infty} \gamma_{x n}^{p}(\theta)\left(\ln \frac{A}{r}\right)^{-n}, \\
\gamma_{y}^{p}=\left(\ln \frac{A}{r}\right)^{\alpha+2} \sum_{n=0}^{\infty} \gamma_{y n}^{p}(\theta)\left(\ln \frac{A}{r}\right)^{-n},
\end{array}\right\}
$$

where

$$
\left.\begin{array}{l}
\gamma_{x 0}^{p}(\theta)=a_{0}, \quad \gamma_{y 0}^{p}(\theta)=b_{0}=c F^{n}, \\
\gamma_{y 1}^{p}(\theta)=-(\alpha+2) c F^{n} \ln (\sin \theta)+\text { const. }
\end{array}\right\}
$$

It is seen from (4.18) and (4.32) that the constant $F$ characterizes the intensity of singularity for the resultant shear stress in plastic zones II and IV (Fig. 1).

We next turn to the determination of the constants $a_{0}, A_{1}, B_{1}$ and angles $\theta_{p}, \theta_{s}$. We define the nondimensional parameter

$$
T=-\frac{F}{G a_{0}(\alpha+1)} .
$$

From the first and the second contiguity conditions (2.14) at the unloading boundary $\Gamma_{\mathrm{B}}\left(\theta=\theta_{p}\right.$, Fig. 1) and the reloading boundary $\Gamma_{\mathrm{D}}\left(\theta=\pi-\theta_{s}\right.$, Fig. 1), respectively, i.e.

$$
\left.\begin{array}{l}
f_{0}\left(\theta_{p}\right)=-F, \quad f_{0}^{\prime}\left(\theta_{p}\right)=0, \\
f_{0}\left(\pi-\theta_{s}\right)=F \sin \theta_{s}, \quad f_{0}^{\prime}\left(\pi-\theta_{s}\right)=-F \cos \theta_{s},
\end{array}\right\}
$$

we obtain

$$
\begin{aligned}
& T=\frac{\pi-\theta_{s}-\theta_{p}}{\cos \theta_{p}}, \\
& \sin \theta_{s} \cdot \exp \left[T\left(1+\sin \theta_{p}\right)\right]-\sin \theta_{p}=0 \\
& A_{1}=\frac{C \cos \theta_{s}+D \cos \theta_{p}}{\sin \left(\theta_{p}+\theta_{s}\right)}, \quad B_{1}=\frac{C \sin \theta_{s}-D \sin \theta_{p}}{\sin \left(\theta_{p}+\theta_{s}\right)},
\end{aligned}
$$

where

$$
\begin{aligned}
& C=T-\sin \theta_{p} \ln \left(\sin \theta_{p}\right)-\left(\frac{\pi}{2}-\theta_{p}\right) \cos \theta_{p} \\
& D=-T \sin \theta_{s}-\sin \theta_{s} \ln \left(\sin \theta_{s}\right)-\left(\frac{\pi}{2}-\theta_{s}\right) \cos \theta_{s}
\end{aligned}
$$

By use of the first of (4.20), (4.24) and (4.36), the first of Eqn. (4.27) can be written in the form,

$$
T\left\{\sin \theta_{p}-S \lambda_{1}^{*}\left(\theta_{p}\right) f_{1}^{* \prime}\left(\theta_{p}\right)\right\}-1=0 .
$$

In the unloading wake zone III, the following unloading condition is required to be satisfied,

$$
K_{0}(\theta) \leqslant F^{2}, \quad \theta_{p} \leqslant \theta \leqslant \pi-\theta_{s} .
$$

Substitution of (4.38) into (4.39) gives $\theta_{s}$ as a function of $\theta_{p}$, which is shown in Fig. 3. Assign an arbitrary value of $S$. Starting from $\theta=0$ with initial condition $f_{1}^{*^{\prime}}(0)=0$, we can integrate 


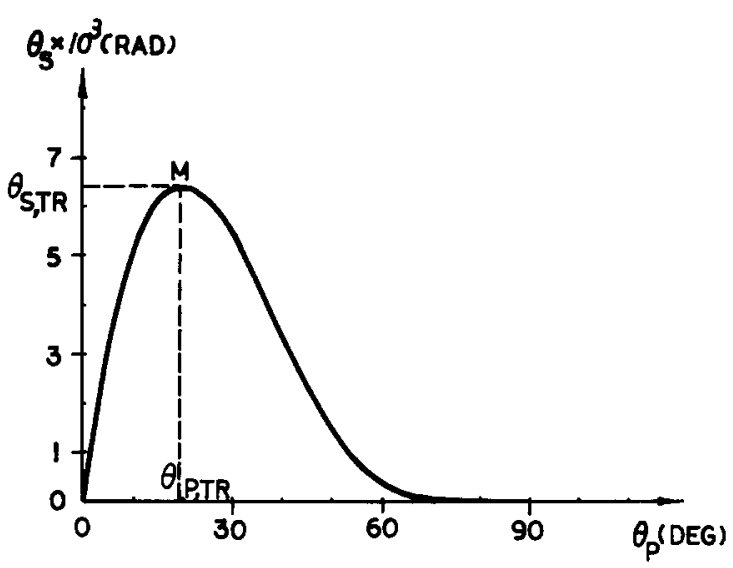

Figure 3. $\vartheta_{s}$ versus $\vartheta_{p}$ curve.

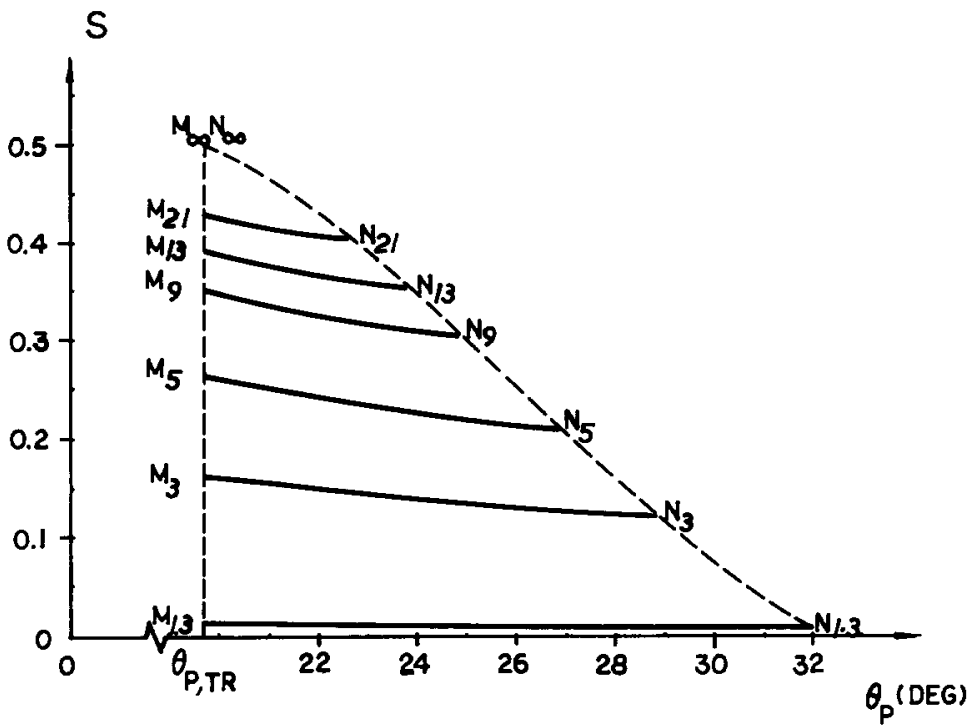

Figure 4. $S$ versus $\vartheta_{p}$ curves for different $n$.

(4.19) to obtain $f_{1}^{*^{\prime}}(\theta)$ and then determine $\theta_{p}$ from (4.41). In Fig. 4 a family of $S$ versus $\theta_{p}$ curves are shown, each corresponding to a parametric value of $n$.

Whatever the value of $n$ may be, there exists the following trivial solution (denoted by the subscript " $t r$ "), which satisfies all the above relations,

$$
\begin{aligned}
& S_{t r}=\frac{1}{n \alpha(\alpha+1)}, \quad T_{t r}=\frac{1}{\sin \theta_{p, t r}}, \\
& f_{1, t r}^{* \prime}(\theta) \equiv 0, \quad \lambda_{1, t r}^{*}(\theta)=n \alpha \cos \theta, \\
& \theta_{p, t r}=0.344026 \operatorname{Rad}=19.7112^{\circ}=19^{\circ} 42^{\prime} 40^{\prime \prime}, \\
& \theta_{s, t r}=0.0063982 \operatorname{Rad}=0.36659^{\circ}=22^{\prime} 00^{\prime \prime} .
\end{aligned}
$$

Here $\theta_{p, t r}$ and $\theta_{s, t r}$ are exactly the angles given by Chitaley and McClintock [1] for elasticperfectly plastic materials $(n \rightarrow \infty)$. In Fig. 3 the trivial solution is denoted by the point $M$, 
which has a maximum value of $\theta_{s}$, and in Fig. 4 by the point $M_{n}$, which varies with $n$. In Fig. 4 the segment $M_{n} N_{n}$ is the possible range of variation of $\left(\theta_{p}, S\right) . M_{n}$ is the extreme state, beyond which the unloading condition (4.42) does not hold true. $N_{n}$ is the other extreme, with its $\theta_{p}$ value denoted by $\theta_{b} . \theta_{b}$ is the maximum boundary for the differential equation (4.19). In other words, the coefficient of the highest derivative term $f_{1}^{* \prime \prime}(\theta)$ vanishes at $\theta=\theta_{b}$,

$$
-n\left\{2 f_{1}^{* \prime}\left(\theta_{b}\right) \sin \theta_{b}-\alpha \cos \theta_{b}\right\}=0 .
$$

Let $\theta_{s b}$ denote the value of $\theta_{s}$ which corresponds to $\theta_{p}=\theta_{b}$. From (4.21),(4.38),(4.41) and (4.44) we obtain the values of $f_{1}^{* \prime}\left(\theta_{p}\right), \lambda_{1}^{*}\left(\theta_{p}\right), T$ and $S$ corresponding to the extreme state $N_{n}$, i.e. for $\theta_{p}=\theta_{b}$

$$
\begin{array}{ll}
f_{1}^{* \prime}\left(\theta_{b}\right)=\frac{1}{2} \alpha \cot \theta_{b}, & \lambda_{1}^{*}\left(\theta_{b}\right)=\frac{1}{2} n \alpha \cos \theta_{b}, \\
T_{b}=\frac{\pi-\theta_{s b}-\theta_{b}}{\cos \theta_{b}}, & S_{b}=\frac{4}{n \alpha^{2}} \tan ^{2} \theta_{b}\left(1-\frac{1}{T_{b} \sin \theta_{b}}\right) .
\end{array}
$$

The values of $\theta_{p}, \theta_{s}$ and $S$ for the extreme states $M_{n}$ and $N_{n}$ are shown in Table 1 for several values of $n$.

TABLE 1

Values of $\theta_{p}, \theta_{s}$ and $S$ for the extreme states $M_{n}$ and $N_{n}$.

\begin{tabular}{lllllllll}
\hline$n$ & $M_{n}$ & & & & & \\
$n$
\end{tabular}

The near-tip stress fields and the resultant stress corresponding to the extreme states $M_{n}$ and $N_{n}$ are shown in Figs. 5 and 6, the near-tip strain fields in Fig. 7. It is clear from Fig. 6 that the unloading condition (4.42) is satisfied. The solution obtained by Chitaley and McClintock for elastic perfectly plastic material results as a limiting case $(n \rightarrow \infty)$ of our solution, when $M_{n} N_{n}$ shrinks to a point.

The stress fields for the trivial solution (i.e. the extreme state $M_{n}$ ) do not depend upon the material. They coincide exactly with those given by Chitaley and McClintock [1] for elasticperfectly plastic materials, whatever the value of $n$ may be. For $n=13$ the two extreme states $M_{n}$ and $N_{n}$ are very near to each other and are hardly distinguishable in Fig. 5 and 6 . As to the strain fields for the trivial solution, they do depend upon $n$.

For the moment in this section we could not determine uniquely the solution, which corresponds to some one state between the extremes $M_{n}$ and $N_{n}$. In the next section we shall show that the $N_{n}$ state is just the true solution.

It should be mentioned that in the solution given in this section the supplementary 


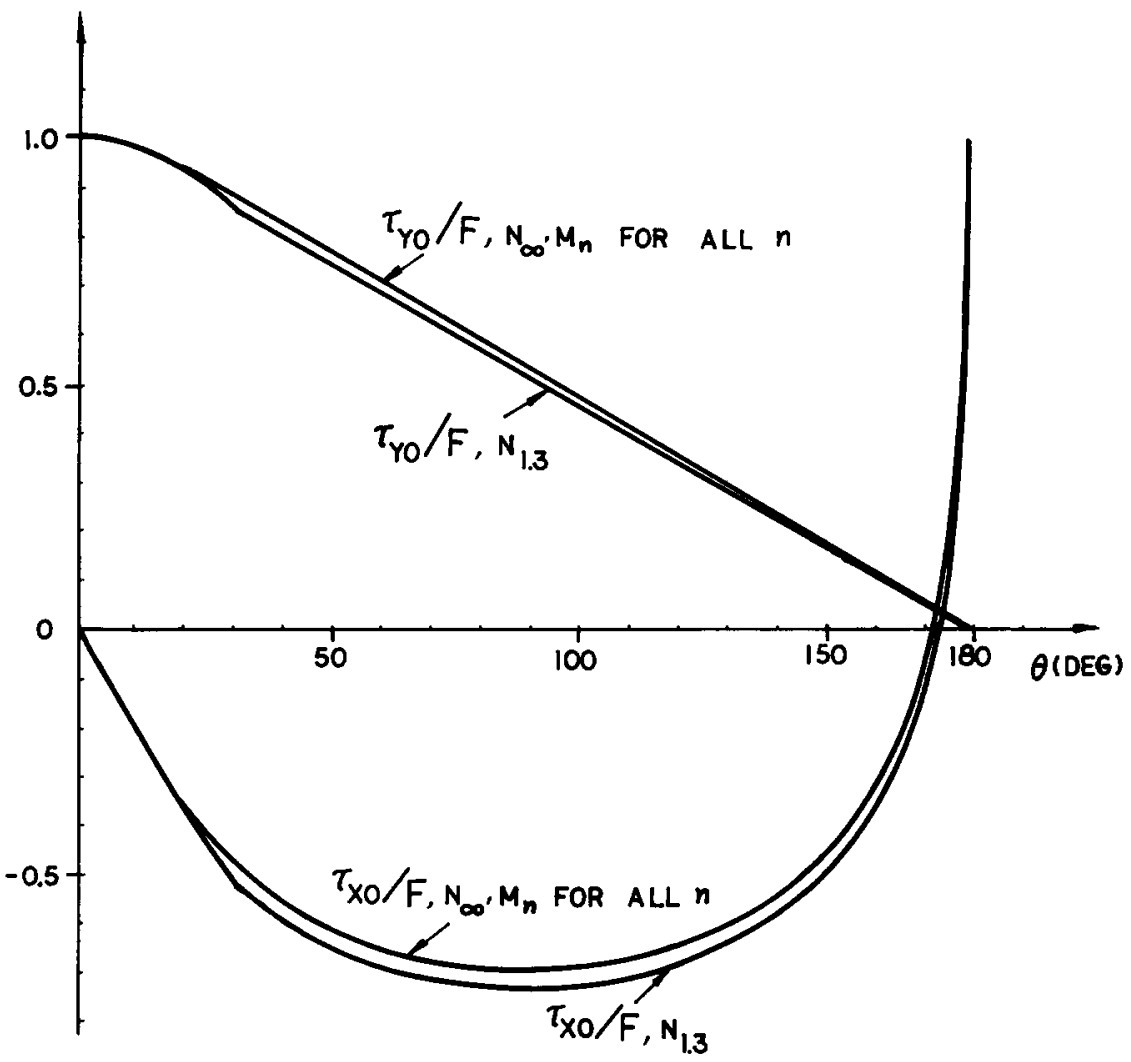

Figure 5. The near-tip stress fields for extreme states $M_{n}$ and $N_{n}$.

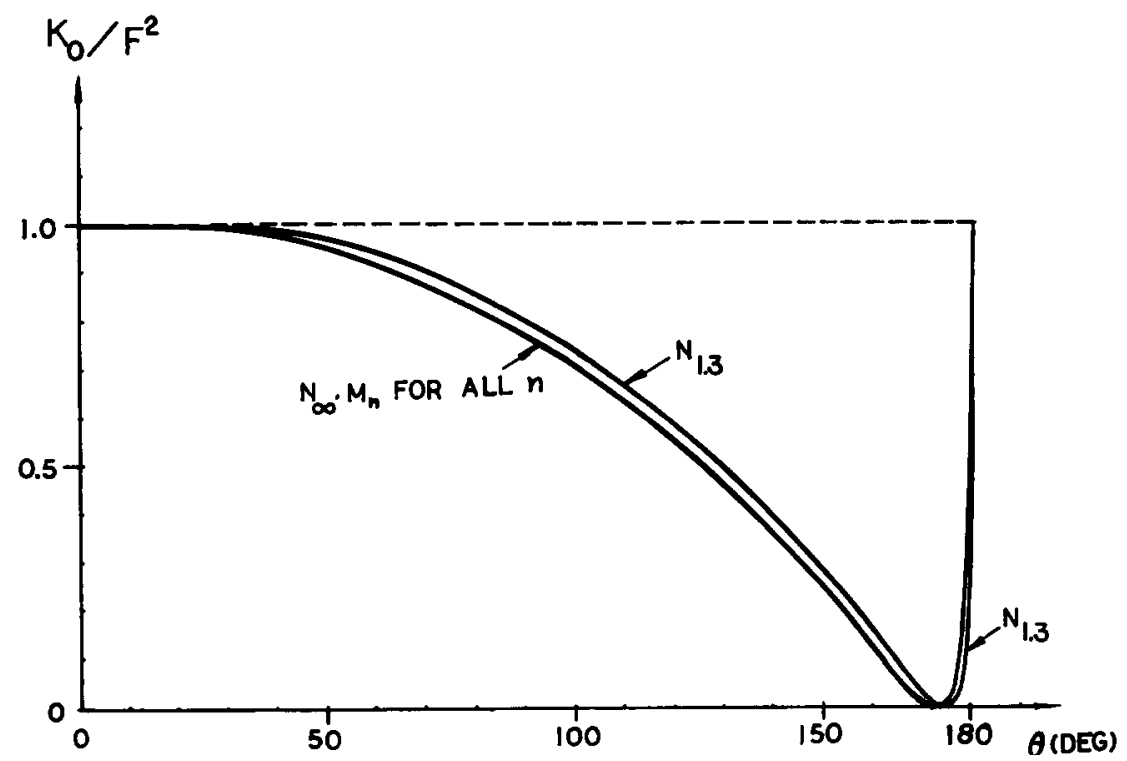

Figure 6. The variation of resultant shear stress for extreme states $M_{n}$ and $N_{n}$. 


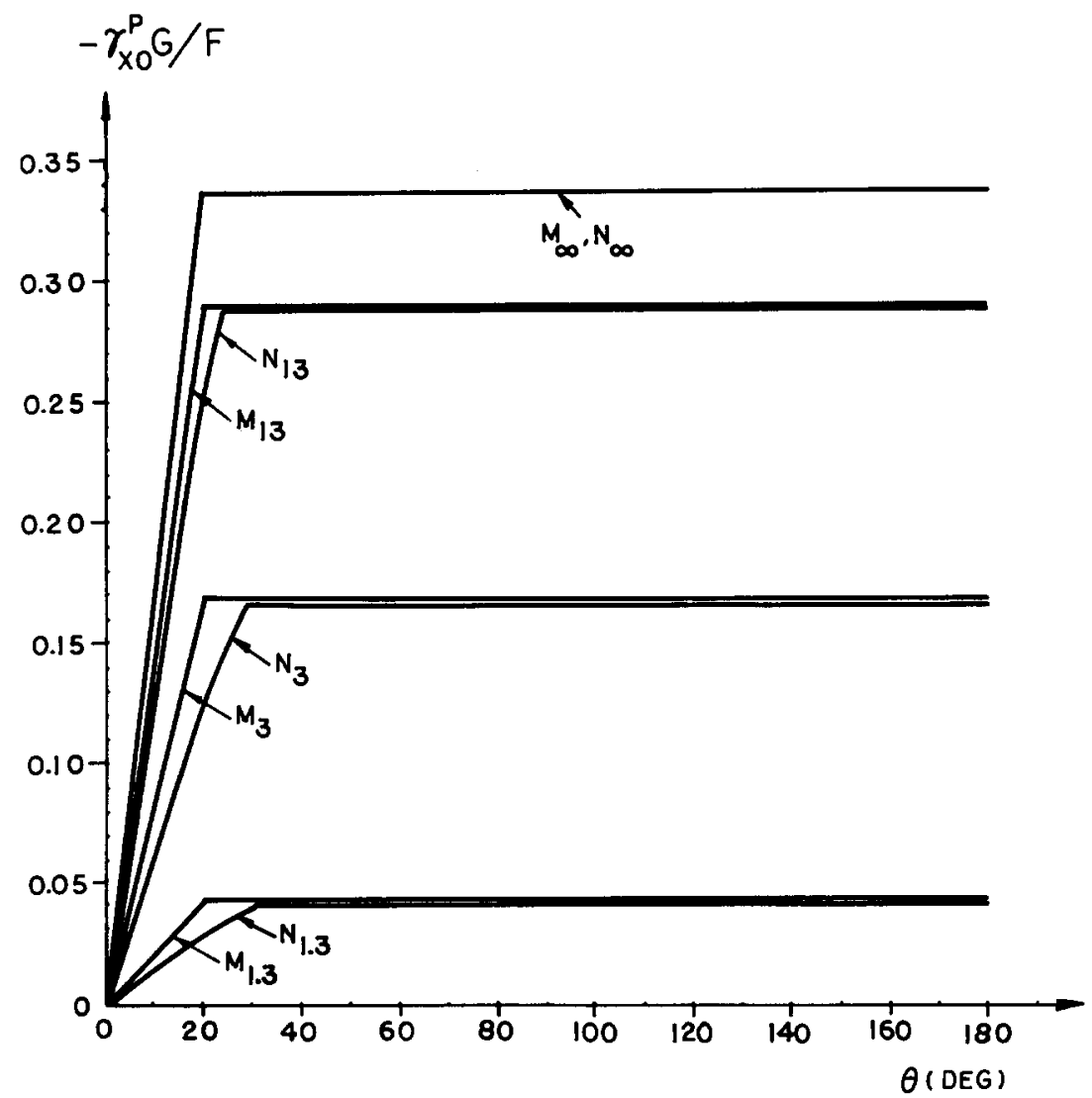

Figure 7. The near-tip strain fields for extreme states $M_{n}$ and $N_{n}$.

condition (2.17) for the unloading boundary $\Gamma_{\mathrm{B}}$, i.e. $\lambda_{1}^{*}\left(\theta_{p}\right)=0$, is not satisfied. We note from (4.44) that

$$
\begin{aligned}
& 2 f_{1}^{* \prime}\left(\theta_{p}\right) \sin \theta_{p}-\alpha \cos \theta_{p}<0, \quad \text { for } \quad \theta_{p, t r} \leqslant \theta_{p}<\theta_{b}, \\
& =0, \text { for } \theta_{p}=\theta_{b} \text {. }
\end{aligned}
$$

It follows then from (4.21) that

$$
\lambda_{1}^{*}\left(\theta_{p}\right)=-n\left\{f_{1}^{* \prime}\left(\theta_{p}\right) \sin \theta_{p}-\alpha \cos \theta_{p}\right\} \geqslant \frac{1}{2} n \alpha \cos \theta_{p}>0, \theta_{p, t r} \leqslant \theta_{p} \leqslant \theta_{b} .
$$

Hutchinson [7] pointed out in comments on the paper [9] that, while $\lambda_{1}^{*}\left(\theta_{p}\right)=0$ is not necessary for the elastic-perfectly plastic problem ${ }^{\star}$, it is a requirement when hardening is present. This deficiency was explained in [10] by the fact that the compatibility equation degenerates from the elliptic to the parabolic type as the crack-tip is approached, and it was predicted that there would be a boundary-layer type solution in the neighborhood of the unloading boundary $\Gamma_{\mathrm{B}}$ (Fig. 1). In the next section we will work out this boundary-layer type solution in more detail.

\footnotetext{
$\star$ We have come to the conclusion in [5] that for elastic-perfectly plastic problem the condition $\lambda_{1}^{*}\left(\theta_{p}\right)=0$ is
} necessary when the unloading boundary does not coincide with the slip line. 


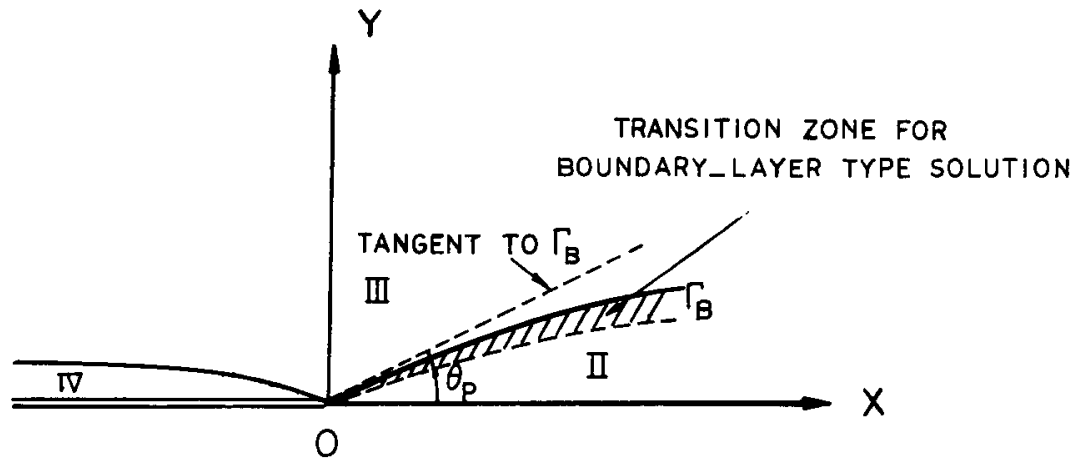

Figure 8. Boundary layer near the unloading boundary $\Gamma_{\mathrm{B}}$.

\section{The inner boundary layer near the unloading boundary}

We assume the equation of the unloading curved boundary $\Gamma_{\mathbf{B}}$ (Fig. 8) to be

$$
\theta=\theta_{p}-\theta^{*}\left(\ln \frac{A}{r}\right)^{-1}
$$

Here the value of the constant $\theta^{*}$ may be either positive or negative. The curve $\Gamma_{\mathrm{B}}$, arbitrarily assumed concave downward in Fig. 8, corresponds to positive $\theta^{*}$. We mention in passing that Dean and Hutchinson [6] have computed the mode III crack for steady growth in elasticperfectly plastic materials, and obtained the unloading boundary, which is concave upward and corresponds to negative $\theta^{*}$.

The solution (4.1) with $f_{0}(\theta)$ and $f_{1}(\theta)$ determined, respectively, by (4.17) and (4.19), denotes the outer solution for zone II (Fig. 8). It is valid or accurate enough outside a narrow transition zone adjacent to the unloading boundary $\Gamma_{\mathbf{B}}$. The outer variables are $r$ and $\theta$. In order to find the boundary-layer type solution, we must use the inner variables $r$ and $\zeta$ instead. Let $\zeta$ be defined as

$$
\zeta=\left(\theta-\theta_{p}\right) \ln \frac{A}{r} .
$$

Then the unloading boundary $\Gamma_{\mathrm{B}}$ is characterized by $\zeta=-\theta^{*}$. We assume the inner solution of the form

$$
\varphi=r\left(\ln \frac{A}{r}\right)^{\alpha}\left\{\psi(\zeta)\left(\ln \frac{A}{r}\right)^{-2}+f_{0}(\theta)+\left(\ln \frac{A}{r}\right)^{-1} f_{1}(\theta)+0\left[\left(\ln \frac{A}{r}\right)^{-2}\right]\right\}
$$

where,

$$
\left.\begin{array}{l}
\psi(\zeta)=\mathrm{o}(\zeta), \\
\frac{\mathrm{d}^{n} \psi}{\mathrm{d} \zeta^{n}}=\mathrm{o}(1), \quad(n \geqslant 1)
\end{array}\right\} \text { as } \zeta \rightarrow-\infty
$$

By use of the transformation (5.2), $f_{0}(\theta)$ and $f_{1}(\theta)$ appearing in (5.3) can be expressed in terms of inner variables as 


$$
\begin{aligned}
& f_{0}(\theta)=f_{0}\left(\theta_{p}\right)+f_{0}^{\prime}\left(\theta_{p}\right) \zeta\left(\ln \frac{A}{r}\right)^{-1}+0\left[\zeta^{2}\left(\ln \frac{A}{r}\right)^{-2}\right] \\
& f_{1}(\theta)=f_{1}\left(\theta_{p}\right)+0\left[\zeta\left(\ln \frac{A}{r}\right)^{-1}\right] .
\end{aligned}
$$

From (5.3) and noting (4.17), i.e. $f_{0}(\theta)=-F$ for zone II, we can obtain immediately the inner solution for the stresses $\tau_{r}, \tau_{\theta}$, and then from (2.10), (2.13), for $\lambda$,

$$
\begin{aligned}
\tau_{r} & =\frac{\partial \varphi}{r \partial \theta}=\left(\ln \frac{A}{r}\right)^{\alpha-1}\left\{f_{1}^{\prime}\left(\theta_{p}\right)+\frac{\mathrm{d} \psi}{\mathrm{d} \zeta}+0\left[\left(\ln \frac{A}{r}\right)^{-1}\right]\right\} \\
\tau_{\theta} & =-\frac{\partial \varphi}{\partial r}=\left(\ln \frac{A}{r}\right)^{\alpha}\left\{F-\left[f_{1}\left(\theta_{p}\right)+\alpha F\right]\left(\ln \frac{A}{r}\right)^{-1}-\psi\left(\ln \frac{A}{r}\right)^{-2}+\right. \\
& \left.+0\left[\left(\ln \frac{A}{r}\right)^{-2}\right]\right\} \\
\lambda & =\frac{n c}{r}\left(\ln \frac{A}{r}\right) F^{n-3}\left\{\left[f_{1}^{\prime}\left(\theta_{p}\right)+\frac{\mathrm{d} \psi}{\mathrm{d} \zeta}\right]\left(-F+\frac{\mathrm{d}^{2} \psi}{\mathrm{d} \zeta^{2}}\right) \sin \theta_{p}+\right. \\
& \left.+\alpha F^{2} \cos \theta_{p}+0\left[\left(\ln \frac{A}{r}\right)^{-1}\right]\right\} .
\end{aligned}
$$

The first asymptotic expansion of the compatibility equation (2.9) can be obtained after a lengthy calculation,

$$
\begin{aligned}
& n c F^{n-3} \frac{\mathrm{d}^{2} \psi}{\mathrm{d} \zeta^{2}}\left\{\sin \theta_{p}\left[f_{1}^{\prime}\left(\theta_{p}\right)+\frac{\mathrm{d} \psi}{\mathrm{d} \zeta}\right]\left(-F+\frac{\mathrm{d}^{2} \psi}{\mathrm{d} \zeta^{2}}\right)+\alpha F^{2} \cos \theta_{p}\right\}+ \\
& +n c F^{n-3} \sin \theta_{p}\left[f_{1}^{\prime}\left(\theta_{p}\right)+\frac{\mathrm{d} \psi}{\mathrm{d} \zeta}\right]\left\{\frac{\mathrm{d}^{2} \psi}{\mathrm{d} \zeta^{2}}\left(-F+\frac{\mathrm{d}^{2} \psi}{\mathrm{d} \zeta^{2}}\right)+\right. \\
& \left.+\left[f_{1}^{\prime}\left(\theta_{p}\right)+\frac{\mathrm{d} \psi}{\mathrm{d} \zeta}\right] \frac{\mathrm{d}^{3} \psi}{\mathrm{d} \zeta^{3}}\right\}+\frac{\sin \theta_{p}}{G} \frac{\mathrm{d}^{3} \psi}{\mathrm{d} \zeta^{3}}=\mathbf{0} .
\end{aligned}
$$

We define

$$
\Psi(\zeta)=\frac{1}{F}\left[\frac{\mathrm{d} \psi}{\mathrm{d} \zeta}+f_{1}^{\prime}\left(\theta_{p}\right)\right]
$$

Then (5.8) can be reduced to

$$
\frac{1}{n S} \Psi^{\prime \prime}(\zeta)+\left[\Psi^{2}(\zeta) \Psi^{\prime}(\zeta)\right]^{\prime}-\left[\Psi^{2}(\zeta)\right]^{\prime}+\alpha \cot \theta_{p} \Psi^{\prime}(\zeta)=0
$$

where $^{\prime}=\mathrm{d} / \mathrm{d} \zeta$. Letting $\zeta \rightarrow-\infty$ in (5.9) and noticing (4.20) and (5.4), we obtain

$$
\Psi(-\infty)=f_{1}^{* \prime}\left(\theta_{p}\right), \quad \Psi^{\prime}(-\infty)=0 .
$$

Integrating (5.10) and noticing (5.11), we get

$$
\Psi^{\prime}(\zeta)\left[\frac{1}{n S}+\Psi^{2}(\zeta)\right]=\left[\Psi(\zeta)-f_{1}^{*^{\prime}}\left(\theta_{p}\right)\right]\left[\Psi(\zeta)+f_{1}^{*^{\prime}}\left(\theta_{p}\right)-\alpha \cot \theta_{p}\right]
$$

We distinguish the two cases in compliance with (4.46) or (4.47).

1. The first case, $\theta_{p} \neq \theta_{b}$, i.e. $\theta_{p, \text { tr }} \leqslant \theta_{p}<\theta_{b}$. Then it follows from (4.46) that $2 f_{1}^{* \prime}\left(\theta_{p}\right)$ $-\alpha \cot \theta_{p}<0$, and the two factors in the right-hand side of (5.12) are different. The solution 
$\Psi(\zeta)$ of (5.12) will be implicitly defined by

$$
\mathrm{e}^{\Psi(\zeta)}\left|\Psi(\zeta)+f_{1}^{*^{\prime}}\left(\theta_{p}\right)-\alpha \cot \theta_{p}\right|^{S_{1}}=C^{*}\left|\Psi(\zeta)-f_{1}^{*^{\prime}}\left(\theta_{p}\right)\right|^{S_{2}} \mathrm{e}^{\zeta}
$$

where $S_{1}$ and $S_{2}$ are constants,

$$
\begin{aligned}
& S_{1}=-\left\{\frac{1}{n S}+\left[f_{1}^{*}\left(\theta_{p}\right)-\alpha \cot \theta_{p}\right]^{2}\right\}\left[2 f_{1}^{*^{\prime}}\left(\theta_{p}\right)-\alpha \cot \theta_{p}\right]^{-1}>0, \\
& S_{2}=-\left\{\frac{1}{n S}+\left[f_{1}^{*^{\prime}}\left(\theta_{p}\right)\right]^{2}\right\} \cdot\left[2 f_{1}^{*^{\prime}}\left(\theta_{p}\right)-\alpha \cot \theta_{p}\right]^{-1}>0 .
\end{aligned}
$$

Letting $\zeta \rightarrow-\infty$ in (5.13) and noticing (5.11), we obtain $1 / C^{*}=0$, and then

$$
\Psi(\zeta)=f_{1}^{*^{\prime}}\left(\theta_{p}\right), \quad \Psi^{\prime}(\zeta)=0 .
$$

2. The second case, $\theta_{p}=\theta_{b}$. It follows from (4.47) that $2 f_{1}^{* \prime}\left(\theta_{b}\right)-\alpha \cot \theta_{b}=0$ and (5.12) becomes

$$
\Psi^{\prime}(\zeta)\left[\frac{1}{n S_{b}}+\Psi^{2}(\zeta)\right]=\left[\Psi(\zeta)-\frac{\alpha}{2} \cot \theta_{b}\right]^{2}
$$

Its integral is

$$
\left|\Psi(\zeta)-\frac{\alpha}{2} \cot \theta_{b}\right|^{\alpha \cdot \cot \theta_{b}} \cdot \exp \left\{\Psi(\zeta)-\frac{\alpha}{2} \cot \theta_{b}^{-1}\right\}=C^{*} \mathrm{e}^{\zeta}
$$

From (5.17) follows the asymptotic behavior of $\Psi(\zeta)$,

$$
\Psi(\zeta)=\frac{\alpha}{2} \cot \theta_{b}-\left(\frac{1}{n S_{b}}+\frac{\alpha^{2}}{4} \cot ^{2} \theta_{b}\right) \frac{1}{\zeta}+o\left(\frac{1}{\zeta}\right), \quad \text { as } \zeta \rightarrow-\infty .
$$

Now we can impose the supplementary condition $\lambda_{1}^{*}\left(\theta_{p}\right)=0$. Equation (5.7) for $\lambda$ can be expressed in terms of $\Psi(\zeta)$ introduced in (5.9) as follows,

$$
\lambda=\frac{n c}{r}\left(\ln \frac{A}{r}\right) F^{n-1}\left\{\Psi(\zeta)\left[-1+\Psi^{\prime}(\zeta)\right] \sin \theta_{p}+\alpha \cos \theta_{p}+0\left[\left(\ln \frac{A}{r}\right)^{-1}\right]\right\} .
$$

Hence, we have

$$
\lambda_{i}^{*}\left(\theta_{p}\right)=n\left\{\Psi\left(-\theta^{*}\right)\left[-1+\Psi^{\prime}\left(-\theta^{*}\right)\right] \sin \theta_{p}+\alpha \cos \theta_{p}\right\} .
$$

For the first case, i.e. $\theta_{p} \neq \theta_{b}$, we obtain from (5.15) and (5.19) nothing other than (4.48), i.e. $\lambda_{1}^{*}\left(\theta_{p}\right)>0$. Therefore, for the first case $\theta_{p} \neq \theta_{b}$, the supplementary condition $\lambda_{1}^{*}\left(\theta_{p}\right)=0$ remains unsatisfied. Therefore we conclude that the true solution is the second case, i.e. $\theta_{p}=\theta_{b}$, which corresponds to the extreme state $N_{n}$ in Fig. 4. The supplementary condition $\lambda_{1}^{*}\left(\theta_{b}\right)=0$ becomes then

$$
\lambda_{1}^{*}\left(\theta_{b}\right)=n\left\{\Psi\left(-\theta^{*}\right)\left[-1+\Psi^{\prime}\left(-\theta^{*}\right)\right] \sin \theta_{b}+\alpha \cos \theta_{b}\right\}=0 .
$$

Putting $\zeta=-\theta^{*}$ in (5.16), solving it simultaneously with (5.20), and noticing the expression for $S_{b}$ in (4.45), we obtain

$$
\Psi\left(-\theta^{*}\right)=\alpha T_{b} \cos \theta_{b}, \quad \Psi^{\prime}\left(-\theta^{*}\right)=1-\frac{1}{T_{b} \sin \theta_{b}} .
$$

We can compute the value of $C^{*} \exp (-\theta)$ from (5.17) by putting $\zeta=-\theta^{*}$. The results are shown in the last column of Table 1 . It can be easily verified that the third contiguity condition 
(2.16), i.e. $\left[\partial^{2} \varphi / \partial n^{2}\right]_{\Gamma_{\mathrm{B}}}=0$, is automatically satisfied. Up to this step we are not able to compute the constants $C^{*}$ and $\theta^{*}$ separately. For the moment we are contented with that due to the consideration of the inner boundary layer we are able to obtain uniquely the asymptotic near-tip solution (i.e. the state $N_{n}$ ) with the plastic part of the strain-rate vanishing at both sides of the unloading boundary $\left(\Gamma_{\mathrm{B}}\right.$ Fig. 1).

\section{REFERENCES}

[1] A.D. Chitaley and F.A. McClintock, Journal of the Mechanics and Physics of Solids 19 (1971) 147-163.

[2] L.I. Slepjan, Mekhanika Tverdogo Tela 9 (1974) 57-67.

[3] Yu-chen Gao, Acta Mechanica Sinica (1980) 48-56 (in Chinese).

[4] J.R. Rice, W.J. Drugan and T.L. Sham, in Fracture Mechanics, A.S.T.M. STP 700 (1980).

[5] Yu-chen Gao and Keh-chih Hwang, in Proceedings IUTAM Symposium on Three Dimensional Constitutive Relationships and Ductile Fracture, Ed. S. Nemat-Nasser (1980).

[6] R.H. Dean and J.W. Hutchinson, in Fracture Mechanics, A.S.T.M. STP 700 (1980).

[7] J.W. Hutchinson, An invited paper to be presented at ICF5, Cannes (1981).

[8] J.C. Amazigo and J.W. Hutchinson, Journal of the Mechanics and Physics of Solids 25 (1977) 81-97.

[9] Yu-chen Gao and Ken-chih Hwang, in Advances in Fracture Research, Vol. 2, Preprints for ICF5, Ed. D. Francois (1981) 669-682.

[10] Yu-chen Gao, Xiao-ti Zhang and Keh-chih Hwang, Acta Mechanica sinica, to be published (in Chinese).

\section{RÉSUMÉ}

On montre que pour une fissure de mode III en croissance stable dans un milieu se consolidant suivant une loi parabolique, la singularité au voisinage de l'extrémité de la fissure ne suit pas une fonction parabolique mais logarithmique. En prenant en considération la couche interne de la frontière qui sépare les zones chargées et les zones non chargées, on obtient des solutions asymptotiques au voisinage de l'extrémité de la fissure pour des champs de contrainte et de déformation; ces solutions satisfont toutes les relations nécessaires de la mécanique des milieux continus en condition élastoplastique, y compris la condition de continuité de vitesse de déformation dans les zones plastiques, lorsque l'on franchit la frontière entre zones chargée et non chargée. 